\title{
A Report of the Women's Health Congress Workshop on The Health of Women of Color: A Critical Intersection at the Corner of Sex/Gender and Race/Ethnicity
}

\author{
Jennifer L. Plank-Bazinet, PhD, Susan G. Kornstein, MD, Janine Austin Clayton, MD, \\ Worta McCaskill-Stevens, MD, MS, ${ }^{3}$ Lauren Wood, MD, Nakela Cook, MD, MPH, \\ Salman M. Tajuddin, MD, PhD, MPH, Gina M. Brown, MD, Tamara Harris, MD, MS, \\ Michele K. Evans, MD, ${ }^{6}$ Lisa Begg, DrPH, RN, ${ }^{1}$ Claudette E. Brooks, MD, Leah R. Miller, PhD, MBA, \\ Amy Caroline Mistretta, MPH, and Terri L. Cornelison, MD, $\mathrm{PhD}^{1}$
}

\begin{abstract}
Women of color face unique health challenges that differ significantly from those of other women and men of color. To bring these issues to light, the National Institutes of Health (NIH) Office of Research on Women's Health sponsored a preconference workshop at the 23rd Annual Women's Health Congress, which was held in Washington, DC, in April 2015. The workshop featured presentations by NIH intramural and extramural scientists who provided insight on the disparities of a wide range of conditions, including cancer, cardiovascular disease, the risk of HIV infection, and disability in an aging population. In this study, we highlight the major points of each presentation and the ensuing discussion.
\end{abstract}

\section{Introduction}

T HERE IS A SHIFT in the demographics of the United States such that in the year 2043 it is predicted to become a majority "minority" nation, with people of color projected to be $53.4 \%$ of the population by $2050 .{ }^{1}$ It is imperative to devote resources to sex/gender and race/ethnicity-based health disparities, especially those faced by women of color. While African American women have a lower incidence of breast cancer, their mortality rate is higher than women from other racial/ethnic groups. ${ }^{2}$ African American and Hispanic/Latina women are more likely to be infected with
HIV than white or Asian women. ${ }^{3}$ There are also significant racial and gender disparities in the onset and outcomes of cardiovascular disease (CVD). ${ }^{4}$ The disparities highlighted here are only a few examples of the specific health concerns faced by women of color.

The Women of Color Health Data Book, fourth edition, provides health and disease data on women by race/ethnicity with relevant discussions of what historical, cultural, and socio-geo-demographic factors may play a role in the health status of women of color and is the seminal publication on this subject. ${ }^{5}$ Health disparities are stratified by race/ethnicity for women; and data on sex differences within various

\footnotetext{
${ }^{1}$ Office of Research on Women's Health, National Institutes of Health, Bethesda, Maryland.

${ }^{2}$ Department of Psychiatry, Institute for Women's Health, Virginia Commonwealth University, Richmond, Virginia.

${ }^{3}$ Community Oncology and Prevention Trials Research Group, National Cancer Institute, National Institutes of Health, Bethesda, Maryland.

${ }^{4}$ Center for Cancer Research, National Cancer Institute, National Institutes of Health, Bethesda, Maryland.

${ }^{5}$ Office of the Director, Division of Cardiovascular Disease, National Heart, Lung, and Blood Institute, National Institutes of Health, Bethesda, Maryland.

${ }^{6}$ Laboratory of Epidemiology and Population Sciences, National Institute on Aging, National Institutes of Health, Baltimore, Maryland.

${ }^{7}$ Office of AIDS Research, National Institutes of Health, Bethesda, Maryland.

${ }^{8}$ Laboratory of Epidemiology, Demography, and Biometry, National Institute on Aging, National Institutes of Health, Baltimore, Maryland.
}

(C) Jennifer L. Plank-Bazinet, et al., 2016; Published by Mary Ann Liebert, Inc. This Open Access article is distributed under the terms of the Creative Commons Attribution Noncommercial License (http://creativecommons.org/licenses/by-nc/4.0/) which permits any noncommercial use, distribution, and reproduction in any medium, provided the original author(s) and the source are credited. 
cultures and people of color are included. These data, disaggregated by sex/gender and race/ethnicity, can be used to observe differences among different groups of women of color. For example, and most germane, in the year 2015, the US Census Bureau projects that African American females will have the shortest life expectancy at birth of any group in the United States. This is a significant concern that current research opportunities are primed to address.

One impediment to fully studying health disparities is the inability to recruit individuals from diverse populations into clinical trials. A 2007 publication highlighted the problem of recruiting African American women to participate in clinical trials and provided guidance for improving recruitment efforts. ${ }^{6}$ The publication indicated common practices to boost participation in clinical trials; most notably were increasing community involvement by the researchers, increasing research directly relevant to African Americans, and ensuring that compensation is sufficient to offset the cost of participating in clinical trials.

On April 16, 2015, the National Institutes of Health (NIH) Office of Research on Women's Health (ORWH), along with The Academy of Women's Health, the Journal of Women's Health, and the Virginia Commonwealth University Institute for Women's Health, sponsored the workshop, The Health of Women of Color: A Critical Intersection at the Corner of Sex/Gender and Race/Ethnicity, as part of the preconference activities of the Women's Health 2015: the 23rd Annual Congress, Washington, DC. This workshop was convened to present the state of the science of healthcare conditions critical for women of color and to generate provocative conversation regarding challenges ahead. The scientists who spoke are members of the NIH intramural and extramural communities, represent multiple $\mathrm{NIH}$ institutes, centers, and offices, and are focused on a wide array of health conditions, including cancer, CVD, HIV infection risk, and disability in an aging population. All presenters emphasized the importance of recruiting diverse clinical trial participants.

The workshop began with a welcome from Dr. Susan Kornstein, Executive Director of the Virginia Commonwealth University Institute for Women's Health, Editor of Journal of Women's Health, and President of the Academy of Women's Health.

\section{Opening Remarks}

Dr. Janine Austin Clayton, NIH Associate Director for Research on Women's Health and Director, ORWH, began the workshop by stressing that life and health scientists have not fully examined the intersection of sex/gender and race/ ethnicity in research studies. As a result, there is much less information about the health disparities of women in general, and women of color in particular, compared with men in general and men of color. When the leading causes of death in US women are examined by race and ethnicity, heart disease, cancer, and stroke are well represented within all groups (African American women, white women, Asian/Pacific Islander women, Hispanic women, and American Indian/Alaska Native women). However, these leading mortality causes vary in order and significance for each group. Adding to this complexity are the findings that many genetically related populations in Africa and the Caribbean display much lower rates of CVD, hypertension, and low birth weight infants, and higher life expectancies than African Americans, casting a doubt on purely genetic explanations for racial health differences. ${ }^{7}$

There are gaps in knowledge about the health outcomes of individuals from underrepresented groups due to a lack of clinical trial data. In some studies, the population has not been sufficiently diverse, while in others, the population has been diverse, but the data have not been analyzed by sex/ gender and race/ethnicity. The lack of diversity in clinical trials data and the lack of disaggregated data analysis impede the translation of discovery into health. In this study, we review some of the recent advances made by NIH scientists.

\section{Presentation Summaries}

\section{Cancer disparities in women of color:} globally and in the United States

Dr. Worta McCaskill-Stevens, Chief, Community Oncology and Prevention Trials Research Group, Division of Cancer Prevention, National Cancer Institute (NCI), delivered a presentation on racial/ethnic disparities in breast cancer incidence, survival, and treatment and also provided an overview of the lack of diversity in clinical trial recruitment and participation.

Breast cancer is the leading type of cancer among women in most parts of the world. ${ }^{8}$ In 2012, there were 1.7 million cases of breast cancer worldwide, which represent $12 \%$ of all cancer cases. Strikingly, the incidence of breast cancer varies greatly by geographical region. ${ }^{8}$ For example, the incidence rate of breast cancer in Western Europe is 89.7 cases per 100,000 individuals, while the incidence rate in East Africa is 19.3 per $100,000 .^{8}$ Despite this lower incidence of breast cancer, the mortality rates in East Africa are higher. Similarly, African American women have a lower incidence of breast cancer, but a higher mortality rate, compared with other American women. $^{2}$

Breast cancer cells may express hormone receptors for estrogen, progesterone, or the human epidermal growth factor receptor 2 (HER2) oncogene. ${ }^{9}$ The overexpression of these receptors on breast cancer cells makes them important targets for breast cancer therapies. ${ }^{9}$ Yet, there are racial/ ethnic differences in the molecular subtypes of breast cancer. Triple-negative breast cancer-negative for estrogen receptors, progesterone receptors, and HER2 - carries a poorer prognosis than hormone receptor-positive breast cancer and has no targeted treatment. This cancer subtype occurs in $27 \%$ of non-Hispanic African American women, but only 14\% of non-Hispanic white women, ${ }^{10}$ which may contribute to the higher mortality rate observed in African American women. ${ }^{2}$ Triple-negative disease does respond to cytotoxic therapy, but it is critical that patients get therapy immediately. ${ }^{11}$

In addition to racial disparities in cancer subtype, there are disparities in clinical trial enrollment and participation that may arise from issues regarding obesity, comorbidity, language barriers, and participant perception. For example, many cancers are related to obesity, which varies greatly by race/ethnicity; 58\% of African American women are obese compared with $33 \%$ of white women. ${ }^{12}$ Furthermore, African American patients may have more comorbidities than white patients. ${ }^{13}$ Obesity and other comorbidities are often exclusion criteria for clinical trials, resulting in underrepresentation of African American women. It is difficult to generalize 
the results of a clinical trial unless all women are eligible to be included.

Language barriers are another issue associated with clinical trial inclusion. Language barriers may prevent Hispanic volunteers from participating in a clinical trial. Hispanic populations in the 100 largest metropolitan areas grew $145 \%$ between 1980 and 2000, indicating the importance of including this population. ${ }^{14}$ Furthermore, language differences may influence how patients interact with providers and the healthcare system, resulting in health disparities.

Lack of diversity in clinical trial participation may be due to participant perception regarding laboratory tests or medical interventions. For example, genetic testing among racial/ethnic minorities is underutilized. ${ }^{15,16}$ Patients are often confused about the difference between germ line and somatic testing, and some individuals are concerned about the harms of germ line screening. African Americans are more concerned about the harms of genetic testing than white Americans, but are just as likely to participate in predictive, prognostic, and pharmacogenomic testing. Therefore, concerted efforts to recruit African Americans for genetic clinical trials are of the utmost importance.

Finally, there is a concern regarding accurate reporting of race/ethnicity. It is recommended that the patient provide this demographic information and that it should not be determined by the provider.

\section{Advances and challenges in the clinical translation of therapeutic cancer vaccines}

Dr. Lauren Wood, Staff Clinician, Vaccine Branch Center for Cancer Research, NCI, oversees translational research for vaccines developed by NCI investigators that are being testing in humans for the first time. She presented information related to the $T$-cell receptor gamma (TCR $\gamma)$ alternative reading frame protein (TARP) vaccine for prostate cancer.

The key function of the immune system is to distinguish self from nonself. The immune system sees self as not dangerous and nonself as dangerous. When self is seen as dangerous, autoimmune diseases arise. Conversely, when nonself is seen as safe, infections such as hepatitis $\mathrm{C}$, human papillomavirus, and tuberculosis can occur. The principle of self and nonself provides an inherent challenge in developing a cancer vaccine because the immune system sees the tumor as self. This makes it difficult for the immune system to attack cancer cells, even though many tumors contain mutations and are not identical to normal tissue. Effective vaccines for cancer must have better antigens so that the immune system recognizes the tumor as something to be attacked. However, tumors can evolve to evade eradication by drugs and they produce molecules that put the brakes on the immune system to prevent it from attacking the tumor. Fortunately, there are checkpoint inhibitors that allow the immune system to overcome the brakes put on by the tumor.

One downside to the use of immunotherapy is that stimulating the immune system can create significant inflammation as the tumor is targeted. As a result, patients often seem to get worse before their condition improves. Furthermore, immunotherapies are more effective in patients with minimal tumor burden. Finally, a lack of reliable, validated surrogate markers for clinical responses makes it difficult to determine the efficacy of immunotherapy as a treatment for cancer.
TARP is a novel protein expressed in more than $90 \%$ of prostate cancers, both primary and metastatic, and $\sim 50 \%$ of breast cancers. ${ }^{17,18}$ It is also expressed in prostate cancers with a range of Gleason patterns and in both hormone-sensitive and castrate-resistant prostate cancer, suggesting that TARP may be an ideal candidate for a novel therapeutic vaccine in any stage of prostate cancer and possibly breast cancer. In a Phase I clinical trial, men with primary prostate cancer were given the TARP vaccine to determine if the vaccine slows the rate of increase in prostate-specific antigen (PSA) levels, an indicator of risk of death from prostate cancer. So far, the results of the trial are promising; the vaccine is safe, and it slows the rise of PSA levels in $72 \%$ of the men who received the vaccine. ${ }^{19}$ However, a key challenge is identifying biomarkers to predict which patients will respond to the vaccine. Studies on the vaccine are ongoing, and a random control trial with a placebo should be completed in 2016.

Cancer immunotherapies represent the epitome of precision medicine. This method harnesses the patient's own cells by genetically engineering them. The advent of immunotherapies means that it is especially important to obtain information on tumor characteristics of all racial/ethnic groups and both sexes/ genders.

\section{Cardiovascular risk and treatment at the intersection of race/ethnicity and sex}

Dr. Nakela Cook, Chief of Staff, Office of the Director, National Heart, Lung, and Blood Institute (NHLBI), presented data on the differences in CVD burden and outcome by sex/gender and race/ethnicity. She also discussed gender differences in several known treatments for CVD.

Several compelling points highlight the importance of bridging the gap in CVD prevention and treatment as it relates to women in general and women of color in particular. Women represent half of all patients with CVD, and CVD is the leading cause of death among women. However, the clinical presentation of CVD can differ between women and men, resulting in symptoms being under-recognized among women. Subsequent underdiagnosis of CVD in some is complicated by gender disparities in the application of evidence-based therapies. Women have traditionally been underrepresented in cardiovascular clinical trials, which can lead to unintended consequences of ambiguity in whether women benefit from therapies tested and proven in predominantly male populations.

There are known differences between the sexes in the pathobiology of CVD. Women develop CVD later in life, probably due to the protective role of hormones when they are younger and the fact that women, on average, live longer than men. There are also certain conditions that affect women more, such as Takotsubo cardiomyopathy, a stress-related cardiomyopathy, in which $90 \%$ of those affected are women. The Women's Ischemia Syndrome Evaluation study evaluated another CVD condition more prevalent among women, myocardial ischemia in the setting of nonobstructive coronary artery disease, and observed a correlation with a higher risk of recurrent cardiac events. ${ }^{20}$

Disparities in CVD manifest in many different facets of the disease. Two studies, Coronary Artery Risk Development in Young Adults (CARDIA) and Atherosclerosis Risk in Communities Surveillance (ARIC), examined disparities in 
heart failure by race/ethnicity and sex/gender. The CARDIA study found that heart failure before the age of 50 is significantly more likely in black Americans than white Americans. This increase in heart failure incidence is likely due to untreated risk factors that occurred earlier in life. ${ }^{21}$ The ARIC study demonstrated that black Americans have the highest overall incidence of heart failure and that black women over the age of 60 are the most likely of any group to experience heart failure. ${ }^{4}$ Women have also been found to be less likely to receive an implantable cardioverter defibrillator (ICD), a life-saving intervention in patients with severe cardiomyopathy, with African American women being the least likely among those eligible to receive one. ${ }^{22,23}$ One study examining trends in ICD utilization from 1999 through 2005, demonstrated a fourfold increase in the 1-year cumulative incidence of ICD use among men. Although the pattern was similar among women, absolute rates of ICD use were $~ 75 \%$ lower than those for men at each point for each entry cohort. ${ }^{24}$ Overall, the data from these large studies indicate that significant disparities exist in regard to heart failure, and women of color are most disproportionately affected.

One of the challenges in addressing the gaps in care for women is that the information regarding treatments for women with CVD is not always complete, and sex-specific studies may be needed to further inform these issues. If women are underrepresented in studies, clinical trial findings may leave uncertainty with regard to implications of the findings for the care of women. For example, glycoprotein IIb/IIIa inhibitors, a proven therapy in the setting of percutaneous coronary intervention for acute myocardial infarction, were shown to produce higher rates of bleeding in women compared with men, raising questions about the riskbenefit profile of this treatment for women. ${ }^{25}$ When sex differences were carefully examined, a substantial amount of the risk of bleeding complications was due to excessive dosing. ${ }^{26}$ Similarly, trials proving the efficacy and effectiveness of ICDs had low representation of women, resulting in uncertainty as to whether women would benefit from their use. A subsequent examination that was focused on women found that women have a substantial mortality benefit from ICDs and that the device therapy should be offered to all eligible patients. ${ }^{27}$

Barriers to including women in clinical trials still remain; eligibility criteria often focus on symptoms, etiology, or pathophysiology that are more common in men. Women develop CVD later in life and thus risk being excluded from trials because they are older, frailer, or have more comorbidities. Underdiagnosis of CVD can also result in a lower likelihood of referral for more cardiovascular testing, which can lead to inadvertently excluding women from trial enrollment depending upon eligibility criteria. Pregnancy concerns and family responsibilities can also pose barriers to inclusion of women. Yet, despite these barriers, it is essential that women participate in clinical trials to ensure sex/gender equity in treatment.

\section{Genome-wide analyses of carotid intima-media thickness among African Americans}

Dr. Salman M. Tajuddin, a postdoctoral fellow in the Laboratory of Epidemiology and Population Sciences, Health Disparities Research Section, National Institute on Aging
(NIA), continued in the topic area of CVD and presented data on single-nucleotide polymorphisms (SNPs) that are associated with carotid intima-media thickness (IMT) and atherosclerosis.

One intermediate marker of CVD is carotid IMT, a measure of subclinical atherosclerosis that strongly predicts future cardiovascular events and is a moderately heritable trait with $31 \%-41 \%$ of the variation in IMT being genetic. ${ }^{28,29}$ Studies of individuals of European ancestry have identified four genetic loci associated with IMT. ${ }^{30}$ Dr. Tajuddin described his work, part of the Health Aging in Neighborhoods of Diversity across the Lifespan (HANDLS) study, ${ }^{31}$ to identify genetic susceptibility factors of carotid IMT among African Americans through genome-wide association analyses.

The analysis identified significantly associated mutations in MMAA and CAMTA1 that could be susceptibility markers for atherosclerosis development among African Americans and could be used to identify high-risk individuals (Tajuddin and Evans, unpublished data). MMAA is involved in the translocation of cobalamin into the mitochondrion and could lead to arterial endothelial damage and atherogenesis as a result of excess homocysteine. ${ }^{32,33}$ CAMTAl is linked with plasma phospholipid fatty acid levels in European ancestry populations. ${ }^{34}$ Dr. Tajuddin also found genetic differences between men and women that were associated with carotid IMT (Tajuddin and Evans, unpublished data). The identified mutations may provide insight into the development of subclinical atherosclerosis. The next step is to conduct a large-scale genome-wide meta-analysis of carotid IMT among African Americans and perform functional studies to identify deleterious effects of significantly associated variants. The information will be used to develop predictive risk scores of those who are likely to develop atherosclerosis.

\section{Sexual violence and the biomedical risk for HIV infection in women}

Dr. Gina M. Brown, Coordinator of Women and Girls and Microbicides Research, Office of AIDS Research, Office of the Director, NIH, discussed the association of sexual violence and the risk for HIV infection and presented startling statistics on HIV infection rates.

Nationally, in 2012, the rate of diagnoses of HIV infection was 18.4 per 100,000 , but that statistic does not show the heavy concentration of the epidemic in some areas of the country. For example, the 2012 HIV infection rate in Washington, DC, was 1,281 per 100,000 women compared with 7.7 per 100,000 women nationwide. Nationwide, 40 per 100,000 black women have HIV; in Washington, DC, the rate was 2,316 per 100,000. The nationwide infection rate for Hispanic women was 7.9 per 100,000, while the rate in the District was 505 per 100,000.

Nationally, African American and Hispanic/Latina women together accounted for estimated $79 \%$ of new HIV cases among females in 2012. ${ }^{3}$ African American women represented $64 \%$ of all new HIV infections among women in 2012 , while representing only $7 \%$ of the entire US population and $13 \%$ of the US female population.

The Centers for Disease Control and Prevention defines intimate partner violence as violence perpetrated by a current or former boyfriend, cohabiting partner, husband, or date. ${ }^{3}$ It can include rape, physical violence, sexual violence, stalking, control of reproductive or sexual health, and emotional abuse. 
The term intimate partner violence is used because $64 \%$ of violence against women is perpetrated by an intimate partner, and $92 \%$ of rapes involve an intimate partner or acquaintance of the victim. ${ }^{3}$

According to the 2014 CDC National Intimate Partner Violence Survey, 1.3 million women were raped in the previous year, and one of five women will be raped in their lifetimes. ${ }^{3}$ While the rates of rape do not differ between white or African American women, African American and Hispanic women have a higher prevalence of HIV infection. Additionally, rape is much more common among young women. Among women who are raped, $81 \%$ experience their first rape by the age of $25 .^{3}$ Similarly, the onset of HIV is more common among this group, making this a young woman's infection. Women who are HIV infected are twice as likely to have experienced intimate partner violence compared with the national average. ${ }^{35}$ Additionally, women who experience intimate partner violence have four times the risk of failure to follow their antiretroviral medication regimen. ${ }^{36}$

The reasons for increased HIV incidence among women who have experienced sexual violence may include compromised negotiation skills, such as the inability to negotiate condom use, and engaging in high-risk behaviors, such as having unprotected sex with a partner who has many other partners. One issue that has been much less explored is the biology of HIV risk. A woman's risk during sexual intercourse is significantly greater than the risk for men as a woman's reproductive tract is contiguous from the vagina to the ovaries, and all of the cells of the reproductive tract can be infected. ${ }^{37}$

Recent research on factors that can influence HIV risk includes studies on how sex and semen together can increase the risk of infection. Sex and semen can increase inflammation and alter the vaginal microbiota; vaginal microflora and inflammation can increase the risk for HIV infection. Moreover, endogenous hormones also can increase the HIV infection risk. There may be a window of increased vulnerability to HIV during pregnancy and ovulation due to hormone-mediated vaginal immune responses.

Future research to better understand the factors that can influence HIV risk should include female reproductive tract biology and how tissue damage from sexual violence can affect risk. Research on genital tract protective factors is also needed.

\section{Disability outcomes: data from the Health, Aging, and Body Composition study}

Dr. Tamara Harris, Chief, Interdisciplinary Studies of Aging Section, Laboratory of Epidemiology and Population Science, NIA, presented information on the role of body composition on disability status. The data were from the Health, Aging, and Body Composition (Health ABC) study, which is a 15-year study consisting of 3,075 participants aged between 70 and 79 years at the start of the study. Forty-two percent of the participants were African American. To be included in the study, participants needed to report the ability to walk a quarter mile and up 10 steps without difficulty at the onset of the study.

One common misconception of the elderly population is that it is homogeneous. In actuality, it is quite heterogeneous and can be divided into three groups: healthy and at low risk for disability, typical with the usual ailments and mobility disability, and frail with more health problems and disability. Those who are frail contribute the most to knowledge about health outcomes, while much less is known about the typical and healthy groups.

NIA is studying the risk factors that underlie the health trajectories of old age. In general, health begins to decline in people's late 70 s or early $80 \mathrm{~s}$, when they might have health incidents that lead to incomplete recovery. This cycle can lead to functional limitations and new episodes of health problems leading to disability.

The Health ABC study hypothesized that a change in body composition is a common pathway by which weight-related health conditions contribute to the risk of disease. African American men and women in the Health ABC study have more subclinical disease compared with white men and women. African American men and white men had about the same average body-mass index (BMI), although African American men had lower fat percentages and more lean mass on average. African American women had a higher average BMI than white women, but more lean mass on average.

In the first 13 years of the ABC study, $80 \%$ of African American women and $73 \%$ of African American men developed functional limitation compared with $72 \%$ of white women and $67 \%$ of white men. The risk factors for functional limitation included lower education level, greater number of hospitalizations, more years of smoking, worse self-rated health, higher BMI, greater adipose and less lean muscle tissue, and weaker grip strength. Mediators of functional limitation among African American women were depression, osteoarthritis of the knees, lower extremity arterial disease, coronary artery disease, lack of exercise, increased cystatin $\mathrm{C}$ levels, and greater fasting glucose. Other risk factors were slower gait and difficulty in walking a longer distance. Age was not a risk factor once all of the other risk factors were accounted for. These data suggest that the onset of disability is complicated, and many different factors may play a role.

\section{Discussion and Closing}

The workshop ended with a discussion led by Dr. Terri L. Cornelison, Associate Director for Clinical Research, ORWH. Several discussion points were raised, including determining whether sex/gender or race/ethnicity is more important for disease outcomes. Dr. Cook indicated that it depends on the risk factors and diseases that are being examined. In some cases, there are narrower racial/ethnic or sex/gender gaps. Some differences by sex/gender relate more to biology, while others relate more to sociodemographics. The complex relationship between biology and sociodemographic factors is also true for race/ethnicity. More risk factors, both demographic and biological, are still being discovered in association with sex/gender and race/ethnicity.

Another discussion was based on whether activism around the violence against women movement could be leveraged to prevent HIV infection and intervention at the community level. One method of intervention is the trauma-informed care model, which involves understanding, recognizing, and responding to the effects of trauma. Changing how trauma care is delivered may bring those at risk to the interventions they need. Dr. Brown also recommends increased community education on HIV risk. In many other countries, HIV prevention is closely intertwined with the violence against 
women and social justice for women movements. Leveraging activism around violence against women to reduce HIV risk is logical to pursue in the United States.

A final discussion focused on the problem of enrollment of racial/ethnic minorities in clinical trials and the extent to which referrals affect the problem. Dr. Cook indicated that there is bias in referral, which is weighted toward men, but the referral problem is only one component of the issue of inclusion. While she concedes that there is much work to be done to understand the factors at play, she stressed that some clinical trials have succeeded in achieving full diversity among participants, indicating that it can be done, but it requires strategizing.

Dr. Cornelison gave an overview of the salient points that were presented and discussed to close the meeting.

- Dr. Clayton posed the question about how race/ethnicity and sex/gender affect the health of women of color.

- Dr. McCaskill-Stevens showed that US women of color carry a greater burden of mortality for breast cancer. She pointed out ways to begin to use genomic-driven treatments. She also said that utilization of germ line testing among racial/ethnic minorities is an important next step.

- Dr. Wood explained the immune system and discussed new cancer vaccines being used to treat prostate cancer that may also be useful in breast cancer.

- Dr. Cook elucidated different pathobiological mechanisms of CVD for women versus men, including myocardial ischemia in the setting of nonobstructive coronary artery disease, and pointed out differences in disease burden, treatment, and outcome by sex/gender and race/ethnicity.

- Dr. Tajuddin discussed atherosclerosis and carotid IMT in terms of the SNPs that have been identified as susceptibility factors. These SNPs may give insight into the mechanisms leading to atherosclerosis. He also highlighted HANDLS, which has been a good model of recruitment and retention of racial/ethnic minorities in age-related health disparities research. ${ }^{31,38}$

- Dr. Brown provided statistics of HIV and women of color and placed this information within the context of sexual violence against women. She also showed how the underlying reproductive tract biology may lead to greater risk of HIV infection after sexual violence.

- Dr. Harris discussed weight-related health conditions and risk of disability. Black women are at increased risk of functional limitation.

In conclusion, The Health of Women of Color: A Critical Intersection at the Corner of Sex/Gender and Race/Ethnicity workshop provided a venue to allow established researchers to present data associated with the intersectionality that affects the health of women of color.

\section{Author Disclosure Statement}

No competing financial interests exist.

\section{References}

1. Bernstein R. United States Census Bureau. U.S. Census Bureau projections show a slower growing, older, more diverse nation a half century from now, 2012. Available at: https://www.census.gov/newsroom/releases/archives/ population/cb12-243.html Accessed June 14, 2015.

2. Centers for Disease Control and Prevention. Breast cancer rates by race and ethnicity, 2014. Available at: www.cdc .gov/cancer/breast/statistics/race.htm Accessed June 14, 2015.

3. Black M, Basile K, Breiding M, et al. The National Intimate Partner and Sexual Violence Survey: 2010 summary report. Atlanta, GA: National Center for Injury Prevention and Control Centers for Disease Control and Prevention, 2011.

4. Loehr LR, Rosamond WD, Chang PP, Folsom AR, Chambless LE. Heart failure incidence and survival (from the Atherosclerosis Risk in Communities study). Am J Cardiol 2008;101:1016-1022.

5. Clayton JA, Brooks CE, Kornstein SG. Toward more individualized medicine: Introducing the Women of Color Health Data Book, Fourth Edition. J Womens Health (Larchmt) 2014;23:781-782.

6. Smith YR, Johnson AM, Newman LA, Greene A, Johnson TR, Rogers JL. Perceptions of clinical research participation among African American women. J Womens Health (Larchmt) 2007;16:423-428.

7. Clark R, Adams JH. Moderating effects of perceived racism on John Henryism and blood pressure reactivity in Black female college students. Ann Behav Med 2004;28: 126-131.

8. Blecher E, Chaney-Graves K, DeSantis C, et al. Global cancer facts and figures, 2nd ed. Atlanta: American Cancer Society, 2008.

9. Yersal O, Barutca S. Biological subtypes of breast cancer: Prognostic and therapeutic implications. World J Clin Oncol 2014;5:412-424.

10. Dietze EC, Sistrunk C, Miranda-Carboni G, O’Regan R, Seewaldt VL. Triple-negative breast cancer in AfricanAmerican women: Disparities versus biology. Nat Rev Cancer 2015;15:248-254.

11. André F, Zielinski CC. Optimal strategies for the treatment of metastatic triple-negative breast cancer with currently approved agents. Ann Oncol 2012;23 Suppl 6:vi46-vi51.

12. Staiano AE, Harrington DM, Johannsen NM, et al. Uncovering physiological mechanisms for health disparities in type 2 diabetes. Ethn Dis 2015;25:31-37.

13. Adams-Campbell LL, Ahaghotu C, Gaskins M, et al. Enrollment of African Americans onto clinical treatment trials: Study design barriers. J Clin Oncol 2004;22: 730-734.

14. Suro R, Singer A. Latino growth in metropolitan America: Changing patterns, new locations. Center on Urban \& Metropolitan Policy and The Pew Hispanic Center, The Brookings Institute, Washington, DC, 2002.

15. Quinn GP, McIntyre JQ, Vadaparampil ST. Challenges in recruiting Mexican women for cancer genetics research. J Community Genet 2011;2:43-47.

16. Sheppard VB, Mays D, LaVeist T, Tercyak KP. Medical mistrust influences black women's level of engagement in BRCA 1/2 genetic counseling and testing. J Natl Med Assoc 2013;105:17-22.

17. Wolfgang CD, Essand M, Vincent JJ, Lee B, Pastan I. TARP: A nuclear protein expressed in prostate and breast cancer cells derived from an alternate reading frame of the T cell receptor gamma chain locus. Proc Natl Acad Sci U S A 2000;97:9437-9442.

18. Epel M, Carmi I, Soueid-Baumgarten S, et al. Targeting TARP, a novel breast and prostate tumor-associated antigen, 
with T cell receptor-like human recombinant antibodies. Eur J Immunol 2008;38:1706-1720.

19. Berzofsky JA, Wood LV, Terabe M. Cancer vaccines: 21st century approaches to harnessing an ancient modality to fight cancer. Expert Rev Vaccines 2013;12:1115-1118.

20. Lewis JF, McGorray SP, Pepine CJ. Assessment of women with suspected myocardial ischemia: Review of findings of the Women's Ischemia Syndrome Evaluation (WISE) Study. Curr Womens Health Rep 2002;2:110-114.

21. Bibbins-Domingo K, Pletcher MJ, Lin F, et al. Racial differences in incident heart failure among young adults. $\mathrm{N}$ Engl J Med 2009;360:1179-1190.

22. Curtis LH, Al-Khatib SM, Shea AM, Hammill BG, Hernandez AF, Schulman KA. Sex differences in the use of implantable cardioverter-defibrillators for primary and secondary prevention of sudden cardiac death. JAMA 2007; 298:1517-1524.

23. Hernandez AF, Fonarow GC, Liang L, et al. Sex and racial differences in the use of implantable cardioverterdefibrillators among patients hospitalized with heart failure. JAMA 2007;298:1525-1532.

24. Al-Khatib SM, Hellkamp AS, Hernandez AF, et al. Trends in use of implantable cardioverter-defibrillator therapy among patients hospitalized for heart failure: Have the previously observed sex and racial disparities changed over time? Circulation 2012;125:1094-1101.

25. Fernandes LS, Tcheng JE, O'Shea JC, et al. Is glycoprotein $\mathrm{IIb} / \mathrm{III}$ a antagonism as effective in women as in men following percutaneous coronary intervention?. Lessons from the ESPRIT study. J Am Coll Cardiol 2002;40:1085-1091.

26. Alexander KP, Chen AY, Newby LK, et al. Sex differences in major bleeding with glycoprotein IIb/IIIa inhibitors: Results from the CRUSADE (Can Rapid risk stratification of Unstable angina patients Suppress ADverse outcomes with Early implementation of the ACC/AHA guidelines) initiative. Circulation 2006;114:1380-1387.

27. Kirklin JK, Naftel DC, Kormos RL, et al. Interagency Registry for Mechanically Assisted Circulatory Support (INTERMACS) analysis of pump thrombosis in the HeartMate II left ventricular assist device. J Heart Lung Transplant 2014;33:12-22.

28. Touboul PJ, Hennerici MG, Meairs S, et al. Mannheim carotid intima-media thickness consensus (2004-2006). An update on behalf of the Advisory Board of the 3rd and 4th Watching the Risk Symposium, 13th and 15th European Stroke Conferences, Mannheim, Germany, 2004, and Brussels, Belgium, 2006. Cerebrovasc Dis 2007;23:75-80.

29. Lange LA, Bowden DW, Langefeld CD, et al. Heritability of carotid artery intima-medial thickness in type 2 diabetes. Stroke 2002;33:1876-1881.

30. Bis JC, Kavousi M, Franceschini N, et al. Meta-analysis of genome-wide association studies from the CHARGE consortium identifies common variants associated with carotid intima media thickness and plaque. Nat Genet 2011;43: 940-947.

31. Evans MK, Lepkowski JM, Powe NR, LaVeist T, Kuczmarski MF, Zonderman AB. Healthy aging in neighborhoods of diversity across the life span (HANDLS): Overcoming barriers to implementing a longitudinal, epidemiologic, urban study of health, race, and socioeconomic status. Ethn Dis 2010;20:267-275.

32. Dobson CM, Wai T, Leclerc D, et al. Identification of the gene responsible for the cblA complementation group of vitamin B12-responsive methylmalonic acidemia based on analysis of prokaryotic gene arrangements. Proc Natl Acad Sci U S A 2002;99:15554-15559.

33. Rossi A, Cerone R, Biancheri R, et al. Early-onset combined methylmalonic aciduria and homocystinuria: Neuroradiologic findings. AJNR Am J Neuroradiol 2001;22:554-563.

34. Wu JH, Lemaitre RN, Manichaikul A, et al. Genome-wide association study identifies novel loci associated with concentrations of four plasma phospholipid fatty acids in the de novo lipogenesis pathway: Results from the Cohorts for Heart and Aging Research in Genomic Epidemiology (CHARGE) consortium. Circ Cardiovasc Genet 2013;6: 171-183.

35. Walsh JL, Senn TE, Carey MP. Exposure to different types of violence and subsequent sexual risk behavior among female STD clinic patients: A latent class analysis. Psychol Violence 2012;2:339-354.

36. Machtinger EL, Haberer JE, Wilson TC, Weiss DS. Recent trauma is associated with antiretroviral failure and HIV transmission risk behavior among HIV-positive women and female-identified transgenders. AIDS Behav 2012;16: 2160-2170.

37. Wira CR, Patel MV, Ghosh M, Mukura L, Fahey JV. Innate immunity in the human female reproductive tract: Endocrine regulation of endogenous antimicrobial protection against HIV and other sexually transmitted infections. Am J Reprod Immunol 2011;65:196-211.

38. Ejiogu N, Norbeck JH, Mason MA, Cromwell BC, Zonderman $\mathrm{AB}$, Evans MK. Recruitment and retention strategies for minority or poor clinical research participants: Lessons from the Healthy Aging in Neighborhoods of Diversity across the Life Span study. Gerontologist 2011;51 Suppl 1:S33-S45.

Address correspondence to: Terri L. Cornelison, MD, PhD Office of Research on Women's Health National Institutes of Health 6707 Democracy Boulevard Suite 400 Bethesda, MD 20817

E-mail: cornelit@od.nih.gov 